\section{Repetitive Stimulation of Muscle}

DURING a study of the effects of repetitive stimulation on the intracellular electrical activity of frog sartorius muscle fibres, alterations were noticed in the voltages of the second and subsequent spikes. At the same time, there was a progressive decrease in $t_{1 / 2}$ after-potential following the second and following spikes. All the experiments were performed on the green frog Hyla coerulea at a temperature of $22^{\circ} \mathrm{C}$.

In preliminary experiments, the frequency of stimulation was increased from $10 / \mathrm{sec}$. to $330 / \mathrm{sec}$., which was the maximum frequency the muscle would follow on indirect stimulation. Up to a frequency of approximately 140/sec., spike voltage and duration remained of normal dimensions, and the $t_{1 / 2}$ afterpotential was not appreciably altered after multiple spikes. Above this frequency, important changes were produced in spike voltages and durations, and $t_{1 / 2}$ after-potential. Fig. 1 shows the records obtained in a single muscle fibre with a progressively increasing number of stimuli at a frequency of $200 / \mathrm{sec}$. A single muscle spike is followed by a negative after-potential of 13-17 mV., with a $t_{1,2}$ value of $13-18 \mathrm{~m}$.sec. A second impulse $5 \mathrm{~m}$.sec. later causes a reduction in the spike voltage, an increase in spike duration, and a shortening of the after-potential. As the number of spikes is increased, spike voltage decreases further until approximately the sixth spike, when a constant voltage is maintained for following spikes. At the same time, $t_{1 / 2}$ after-potential decreases until it reaches $6 \mathrm{~m} . \mathrm{sec}$., in this record, after the eleventh spike. Generally, once a $t_{1 / 2}$ of $6 \mathrm{~m}$.sec. had been reached, no more spikes could be elicited. These results were repeated in many fibres, and all showed the same general trend of reduction in spike voltage and $t_{1 / 2}$ after-potential to limiting values which were constant for each muscle studied. Voltage of the negative after-potential did not appreciably alter as the number of stimuli was increased.

Experimental procedures which altered the $t_{1 / 2}$ after-potential, such as varying external concen-

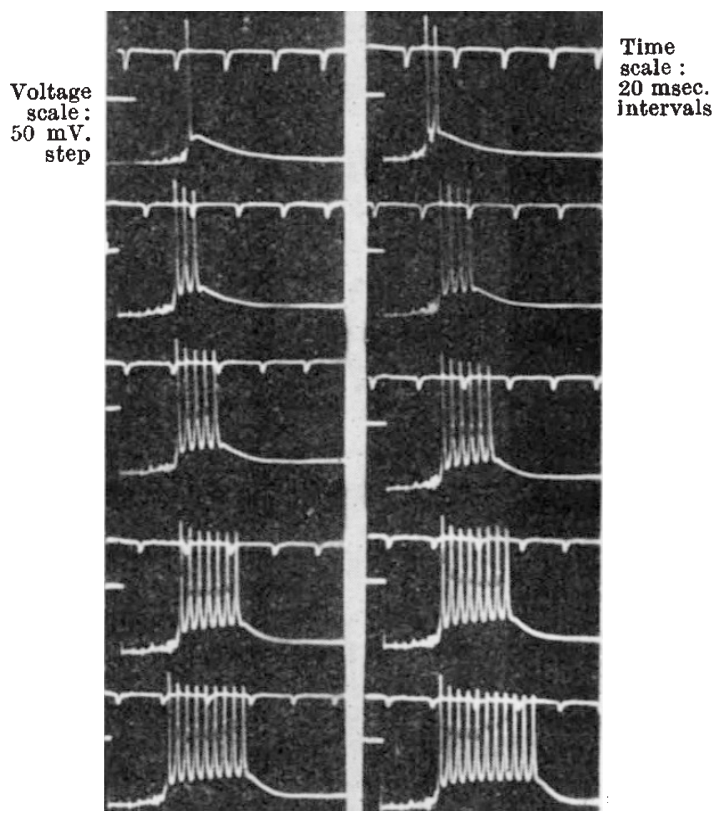

Fig. 1 trations of potassium, sodium and calcium ions, decreased or increased the number of spikes necessary to produce a $t_{1 / 2}$ of $6 \mathrm{~m} . \mathrm{sec}$. In all experimental conditions, a $t_{1 / 2}$ value of $6 \mathrm{~m}$.sec. was reached.

It is thought that the progressive shortening of the after-potential is due to an accumulation of potassium ions immediately outside the muscle fibres.

Publication of details of this work will follow after these preliminary observations have been more fully studied.

Sir William Macgregor School of

M. T. Osborne Physiology,

University of Queensland, Brisbane. March 25.

\section{Electrical Responses of Neuron Cell-Bodies in Sodium-free Media}

THE electrical activity of frog spinal ganglion cells is maintained, and even enhanced, when the external sodium has been totally replaced by equimolar amounts of either choline or tetraethylammonium ${ }^{1}$. Further investigations suggested that quaternary ammonium ions do not actually substitute for sodium as carriers of the action current, but probably exert a pharmacological action on the membrane or in the perikaryon ${ }^{2}$.

A new series of experiments were performed to assess the effects on frog spinal ganglion cells of other related compounds which maintain the excitability of frog nerve fibres ${ }^{3}$ or crab muscle fibres ${ }^{4}$ deficient in sodium. The various solutions used contained, in addition to the amount of the drug tested, $1.8 \mathrm{~m} M$ calcium, $2 \mathrm{~m} M$ potassium, and sucrose to make up isotonicity $(p \mathrm{H} 6 \cdot 4-7 \cdot 0)$.

Sodium-free $112 \mathrm{mM}$ solutions of tetramethylammonium, diethanol-dimethylammonium and acetylcholine chlorides had effects similar to those of a $112 \mathrm{mM}$ choline chloride solution. A sodiumfree 5-10 $\mathrm{m} M$ solution of tetrabutylammonium chloride maintained the excitability of a number of cells, but higher concentrations irreversibly depolarized and blocked them. A sodium-free $112 \mathrm{mM}$ solution of ammonium chloride ( $p \mathrm{H}$ adjusted to $7 \cdot 0$ ) exerted a powerful depolarizing action on the cells, but overshooting and prolonged electrical responses were obtained with $25 \mathrm{mM}$, although the resting potential still had a low value. A sodium-free $112 \mathrm{mM}$ solution of guanidine hydrochloride maintained the excitability of the cells though the latter were slightly depolarized in this medium. A sodium-free $112 \mathrm{mM}$ solution of hydrazine ( $p \mathrm{H}$ adjusted to 6.4) had a very characteristic effect on the cell-body, as compared with the other onium ions. The configuration of the action potentials obtained in this medium was practically indistinguishable from that of responses in normal Ringer. Action potentials could be evoked by 'direct' and also by 'indirect' (that is, by propa. gated nerve impulses) stimulation.

With the exception of hydrazinium ion, the various active onium ions used in sodium-free solutions produced action potentials with a characteristic configuration (namely, peak, plateau, slow falling-phase), different from the 'normal' brief spikes recorded in Ringer's solution. The duration of the electrical responses, more than their peak height or rate of rise, was dependent on the kind and concentration of the drug applied. These prolonged action potentials 